\title{
Structural sequence evolution and Computational Modeling Approaches of the Complement System in Leishmaniasis
}

\author{
Prajakta Ingale ${ }^{1}$, Ritika Kabra and Shailza Singh ${ }^{1^{*}}$ \\ ${ }^{1}$ National Centre for Cell Science, NCCS Complex, Ganeshkhind, Pune 411007, India
}

\begin{abstract}
The complement system acts as central arm of innate immunity that is critical to host defense as well as the development of adaptive immunity. The origins of the complement system have so far been traced, which correlates to near to the beginnings of multi-cellular animal life. Owing to the difficulty in obtaining crystal structures of GPCRs in either inactive or active state, accurate structural modeling is still highly desirable for the majority of GPCRs. In an attempt to dissect the conformational changes associated with GPCR activation, computational modeling approaches is being pursued in this paper along with the evolutionary divergence to deal with the structural variability.
\end{abstract}

Keywords: C5aR1, Leishmaniasis, molecular clock, complement system 


\section{Introduction:}

G-protein-coupled receptors (GPCRs) are the largest and most diverse family of transmembrane receptors. [1] The C5a receptor, known as complement component 5a receptor 1 (C5AR1) is a G protein-coupled receptor for C5a. [2] It is one of the major chemoattractant receptors. To date, $\mathrm{C} 5 \mathrm{aR}$ has only been identified and cloned in mammalian species, and its evolutionary history remains ill-defined. [3,4] A relatively low homology level has been observed across the mammalian C5aR sequences; for example, human C5aR is only 65, 67, 68, and 70.8\% identical with $\mathrm{C} 5 \mathrm{aR}$ from mouse, guinea pig, dog, and rat, respectively.[5] All these C5aR molecules possess an extra- cellular N-terminal region, seven helical hydrophobic TM regions and an intracellular C-terminal domain. C5a interact with receptor protein C5a or C5aR on the surface of target cells such as macrophages, neutrophils and endothelial cells. To gain insights into the evolution and to study the divergence time between the groups of these C5aR receptor proteins, we have carried out the molecular clock analysis.

G-protein-coupled receptors (GPCRs) regulate a diverse range of intracellular signaling pathways mediated mainly by guanine nucleotide binding proteins. These pathways form an intricate network involved in critical biological tasks but not limited to homeostasis and transcriptional factors etc. Given the central role of GPCRs in the overall biology of the cell, they undoubtedly represent the most relevant group of therapeutic targets. As stated the cross-talk network is an intricate means of regulation, synergizing weak stimuli and reigning in collateral damage. Leishmania is already well known to exploit these interactions with a multitude of evasion tactics, working to reduce its detection and prolong its survival. Most of the described mechanisms show the coupling of inhibitory receptors that dampen cellular immunity or misguide the attack. One well described method appropriates the complement system, which is among the first of the parasite host interactions. Seemingly in conflict with the definition of 'evasion' Leishmania of all species, potently and selectively activates complement. This selectivity is found between the various stages of the parasite, whereby promastigotes and amastigotes can differentially target the human complement receptors CR1 and CR3 to suit their distinct living conditions. This cunning use of cross talk increases phagocytosis through opsonized complement receptor mediated uptake and decrease the intensity of the oxidative burst 
within the phagosome. It is so pivotal to pathogenesis that genetic ablation of the complement receptor C5aR renders mice resistant to infection with L. major. [6]

Owing to the difficulty in obtaining crystal structures of GPCRs in either inactive or active state, accurate structural modeling is still highly desirable for the majority of GPCRs. Over the years, structural modeling of GPCRs has mostly been obtained through homology modeling using available crystal structures as suitable templates but various $a b$ initio approaches have also been tested. Computational methods that have been applied in the course of these years to study the dynamical properties of GPCRs in an attempt to dissect the conformational changes associated with their activation, is being pursued in this paper along with the molecular clock analysis.

\section{Materials and methods:}

\subsection{Data Collection:}

Total 22 coding sequences of C5AR1 genes (of different vertebrates) were retrieved form gene database of NCBI in .fasta file format. These .fasta files merge together to form multifasta file. The accession no. of different organism's C5AR1 included for the analysis is given as below;

\begin{tabular}{|c|c|c|c|}
\hline Sr. No. & Accession No. & Scientific Name & Common Name \\
\hline 1. & GI|291167767| & Mus musculus & House mouse \\
\hline 2. & GI|112293258| & Homo sapiens & Human \\
\hline 3. & GI|847158952| & Xenopus tropicalis & Frog \\
\hline 4. & GI|297277451| & Macaca mulatta & Monkey \\
\hline 5. & GI|694975876| & Pan troglodytes & chimpanzee \\
\hline 6. & GI $|545175925|$ & Equus caballus & Horse \\
\hline 7. & GI|16758417| & Rattus norvegicus & Rat \\
\hline 8. & GI|166999226| & Bos taurus & Cattle \\
\hline 9. & GI|50979237| & Canis lupus familiaris & domestic dog \\
\hline 10. & GI|346644763| & Sus scrofa & wild boar \\
\hline 11. & GI|290543381| & Cavia porcellus & Guinea pig \\
\hline 12. & GI|744599290| & Camelus dromedarius & Camel \\
\hline 13. & GI|548505144| & Capra hircus & Goat \\
\hline 14. & GI|591293794| & Panthera tigris altaica & Tiger \\
\hline
\end{tabular}




\begin{tabular}{|l|l|l|l|}
\hline 15. & GI $564240887 \mid$ & Alligator mississippiensis & Alligator \\
\hline 16. & GI $587013626 \mid$ & Felis catus & Cat \\
\hline 17. & GI $655892848 \mid$ & Oryctolagus cuniculus & Rabbit \\
\hline 18. & GI $752416049 \mid$ & Ailuropoda melanoleuca & Giant panda \\
\hline 19. & GI $688598194 \mid$ & Danio rerio & Zebrafish \\
\hline 20. & GI $602652885 \mid$ & Python bivittatus & Python \\
\hline 21. & GI $|675726191|$ & Pan paniscus & Bonobo \\
\hline 22. & GI $|426389371|$ & Gorilla & Gorilla \\
\hline
\end{tabular}

\subsection{Sequence alignment and phylogenetic reconstruction:}

Multiple sequence alignment was performed using standalone clustalW2 program [7] and the .aln file generated was converted to .nexus format to create Bayesian inference phylogeny and molecular clock using MrBayes (Version 3.2.2 x64). [8, 9] The final trees generated by the MrBayes were visualized and edited using Fig tree software (vs 1.4.2). [10]

\subsubsection{Bayesian inference phylogeny and Relaxed Clock Model}

To estimate Bayesian inference phylogeny, GTR $+\mathrm{I}+\mathrm{G}$ model was used and Markov chain Monte Carlo (MCMC) simulation was run for 30,000 generations, until the standard deviation of split frequencies were below 0.01. Parameters and corresponding tree were summarized after discarding the initial $25 \%$ of each chain as burnin. Information regarding the out-groups was obtained using the resulting tree generated by Bayesian inference method. Relaxed molecular clock was constructed using the same dataset by running 4,00,000 generations of MCMC simulation. The model used for constructing the molecular clock was the independent gamma rates model (IGR model).

\subsubsection{Node dating of relaxed molecular clock}

We have calibrated the relaxed clock model based on the node calibration (node dating). Based on the default setting e. g, the clock rate is fixed to 1.0, means the age of the nodes in the tree will be measured in the number of expected substitution per site per million years, we assume that the rate is approximately $0.01 \pm 0.005$ substitutions per site per million year. For this we 
have use 0.02 as the mean and 0.005 as the standard deviation. Since we give these values using millions of years as the unit, the resulting tree will be calibrated in millions of years.

\subsection{Homology Modeling and Molecular Dynamics Study}

The primary amino acid sequence of human C5AR1 was downloaded from NCBI gene sequence database (NP_001727). Visualization and presentation of model structure was performed in Pymol (PyMOL Molecular Graphics System, Version 1.7, Schrödinger, and LLC). Ab-initio modeling was carried out using online I-TASSER server. [11] Loop refinement and quality assessment was performed using MODELLER and also other online server like SAVES, PDBsum [12] RAMPAGE etc. [13, 14] and finally the energy minimization and atomistic simulation study was performed using GROMACS [15].

\subsection{Molecular dynamics simulation of C5aR1}

MD simulation was performed using OPLS-AA (all-atom) force field and the SPC (Simple Point Charge) water model. The protein was solvated with water in periodic cubic box of system size $77.74 \AA \times 51.32 \AA \times 90.78 \AA$ and the minimal distance of $1.0 \mathrm{~nm}(10 \AA)$ was applied between the edge of the box and the protein. First the energy minimization was carried out, which has taken 2329 steps of steepest descent to get converged. After minimization potential energy noted was $-3.8550902 \mathrm{e}+06$. The temperature and pressure was controlled with the v-rescale and Berendsen weak coupling algorithms. Finally the production MD was performed for $12 \mathrm{~ns}$ time steps until the model gets stabilized. After completion of the simulation steps, the trajectory of the protein, energy and RMSD (root-mean-square- deviation), RMSF (root-mean-squarefluctuation) were analyzed.

\section{C5aR1 Lipid Bilayer (POPC) Membrane MD Simulations}

After successful MD simulation minimum energy conformation of C5aR1 structure was obtained from trajectory. C5aR1 belongs to the family of GPCRs; their 7 transmembrane helical domains were identified visually by analyzing the structure. For the Lipid bilayer membrane insertion POPC (1-palmitoyl-2-oleoyl-sn-glycero-3-phosphocholine) [17] was selected due to its versatile vesicle formation ability. Lipid bilayer was inserted using Desmond (DE Shaw Research, USA) software System Builder module. Total 182 POPC molecules were inserted to cover the entire 7 transmembrane helices of C5aR1. The area per lipid of POPC was around $68.2 \AA \square^{2}$. After 
insertion of POPC bilayer TIP3P [18] water model was used for solvation. Other parameters used were kept constant which were mentioned in earlier MD simulation section. A total of 5ns of production MD run was performed with $2 \mathrm{fs}$ of time step and snapshots were saved for every 2ps. At the end of the MD simulation POPC area per lipid was close to experimentally determined areas $\left(68.3 \pm 1.5 \AA \square^{2}\right)$, thus indicating that this lipid bilayer MD simulation is successful. 


\section{Result and Discussion:}

\subsection{Bayesian inference phylogeny and Relaxed clock model:}

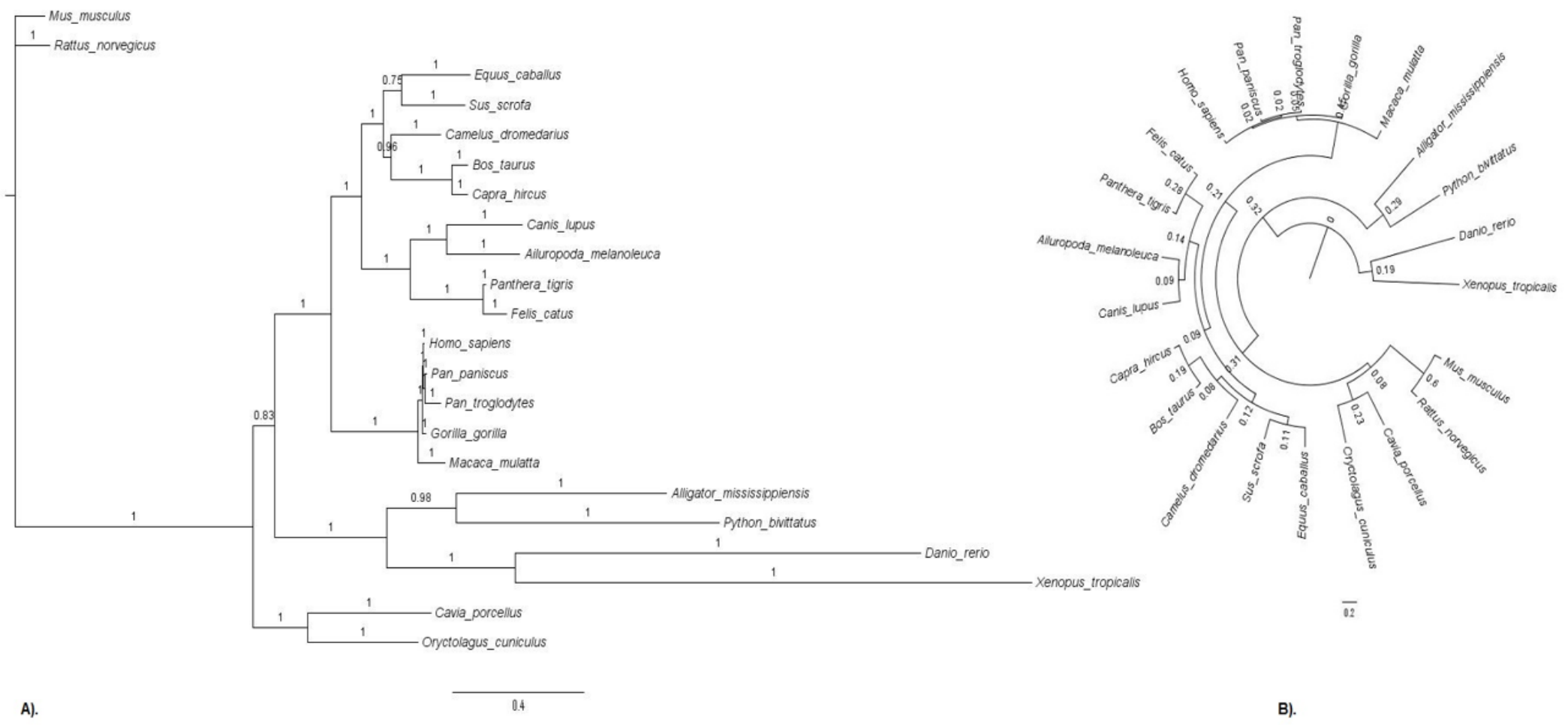

Figure 1: A). Phylogram based on Bayesian analysis of 22 taxa of C5AR1 genes of different organisms. The horizontal lines are branches and represent evolutionary lineages changing over time. The longer the branch in the horizontal dimension, the larger the amount of change. The bar at the bottom of the figure provides a scale for this. In this case the line segment with the number '0.4' shows the length of branch that represents an amount genetic change of 0.4. Support values on branches are Bayesian posterior probabilities (BPPs). A high value means that there is strong evidence that the sequences to the right of the node cluster together to the 
exclusion of any other. The species Ratus norvegicus and Mus musculus were included as out-group. B). Relax clock model based on the Bayesian tree. The support value at each node indicates the branch length that is proportional to time. The scale bar at the bottom of the figure represents the amount of change that is 0.2 . Here we can see that relaxed clock model generated was accelerating with respect to the out-group.

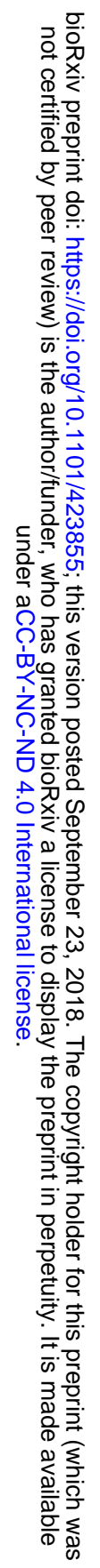




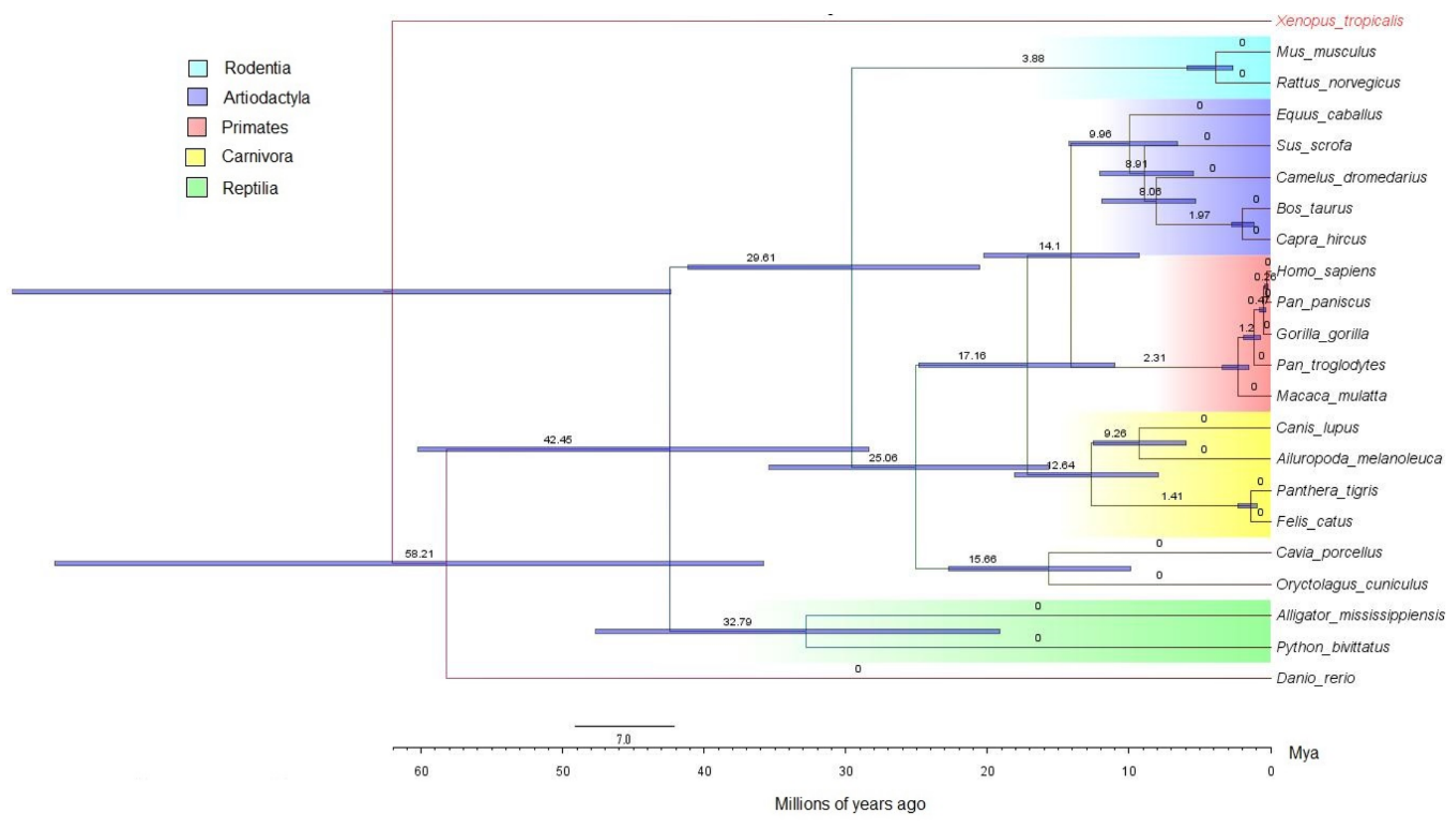

Figure 2: Chronogram of Relaxed clock model based on node dating (ND). Here the node labels indicate the median age (mya) and gray node bars shows the 95\% HDP of the estimated node age. Out-group taxa used to root the tree include Xenopus tropicalis. The clades were marked with different shades of colors corresponding to the distribution of the taxa according to their order. The divergence time is indicated in Millions of years (Mya) below the figure. Scale bar represents the number of substitutions per site. 


\subsection{Molecular Modeling of C5aR1}

In order to carry out homology modeling using MODELLER of C5aR1, best template selection was performed through PSI BLAST of the target protein against the PDB database (http://www.rcsb.org/pdb/home/home.do). The significant hits with $\geq 30 \%$ sequence identity, was selected as templates for the target protein. The blast result shows that 4DKL allow relatively high (30\%) sequence similarity with C5aR1. After generating the homology based model using MODELLER it was observed that the N-terminus region of C5aR1 was not able to span over the entire 4DKL, as a result the secondary structure of initial 34 residue of $\mathrm{N}$-terminus was not obtained. So ab-initio modeling using I-TASSER (Iterative Threading Assembly Refinement) server was performed.

Three criteria (C-Score, TM score and RMSD values) were employed to compare the quality of predicted models to choose the best one. Model 1 was predicted to have a C-Score -0.42 which was comparatively better than the other homology model. The estimated computational TM score $0.66 \pm 0.13$ and RMSD 7.4 \pm 4.3 of model 1 was found to be better than the values obtained for other model.

I-TASSER provides best possible 5 models; we can choose any one out of these 5 structures. This server provides the TM score and RMSD for only first structure although the other structures can also be considered if it fulfills the other criteria. Here, the below table provides the 5 structures generated by I-TASSER and their different parameter values.

Following are the results obtained by I-TASSER server: 


\begin{tabular}{|c|c|c|c|}
\hline I-TASSER & C-Score & TM score & RMSD \\
\hline Model 1 & -0.42 & $0.66 \pm 0.13$ & $7.4 \pm 4.3 \AA$ \\
\hline Model 2 & -1.61 & - & - \\
\hline Model 3 & -3.55 & - & - \\
Model 4 & -3.57 & - & - \\
\hline Model 5 & -3.35 & - & - \\
\hline
\end{tabular}

Table 1: Three criteria (C-Score, TM score and RMSD values) of I-TASSER

After analyzing I-TASSER parameters, these structures were further assessed through Ramachandran plot using RAMPAGE, dope score and GA341 score using MODELLER. The result obtained after the analysis is shown as below:

\begin{tabular}{|c|c|c|c|c|c|}
\hline I-TASSER & \multicolumn{3}{|c|}{ RAMPAGE } & Dope score & GA341 \\
Models & Favored & Allowed & Outliers & & \\
Model 1 & 292 & 40 & 16 & -45573.589844 & 1.000000 \\
Model 2 & 289 & 43 & 16 & -42572.429688 & 1.000000 \\
\hline Model 3 & 267 & 59 & 22 & -44035.679088 & 1.000000 \\
\hline Model 4 & 288 & 38 & 22 & -43878.457031 & 1.000000 \\
Model 5 & 297 & 36 & 15 & -44077.480469 & 1.000000 \\
\hline
\end{tabular}

Table 2: Result of RAMPAGE and MODELLER modules 
Based on above analysis, it can be said that the value of dope score and GA341 score was within the limit but the arrangement of residues based on Ramchandran plot required loop refinement. Visualization of the structure was performed using Pymol. [16]. Model 4 showed the structural correlation with the predefined structure of model C5aR1 as it contains 7 helical domains, 3 intra and 3 extra cellular loops (Figure 3). Initial refinement of 3D model generated was carried out with the help of loop refinement protocol of MODELLER. For this script loop.py was run in order to move 22 residues from the outliers to allowed region. The final structure obtained was again analyzed for its quality assessment.

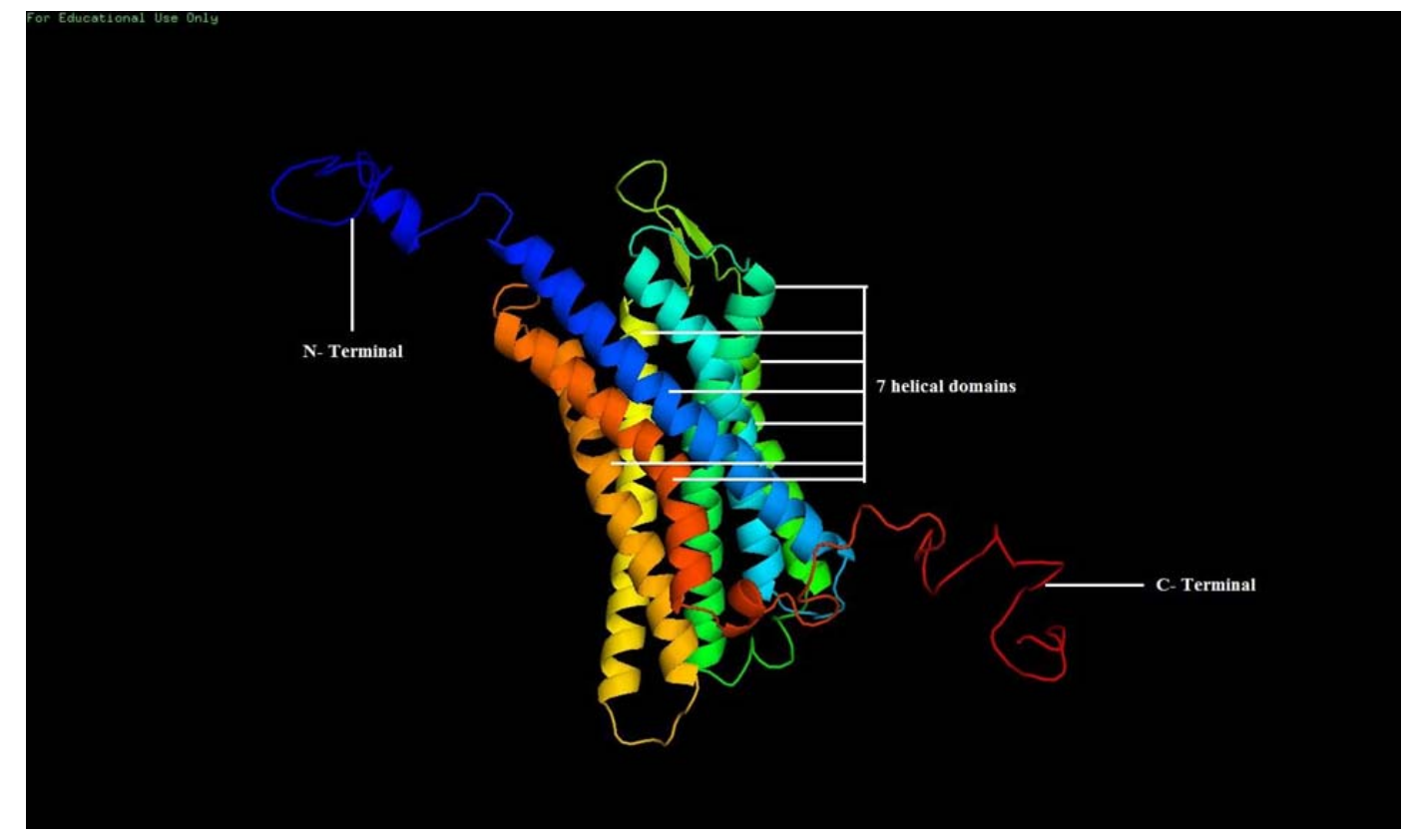

Figure 3: Final model (model 4) of C5aR1.pdb visualized through pymol. Here initial blue part represents the free N- terminal region where as red part shows the free $\mathrm{C}$ - terminal region, the middle region has 7 helical domains, showing resemblance with the predetermined theoretical model. 


\begin{tabular}{|c|c|c|c|}
\hline \multirow{3}{*}{$\begin{array}{c}\text { I-TASSER } \\
\text { Final model } \\
\text { 4.pdb }\end{array}$} & \multicolumn{3}{|c|}{ RAMPAGE } \\
\hline & Favored & Allowed & Outliers \\
\hline & 322 & 26 & 0 \\
\hline Dope score & \multicolumn{3}{|c|}{-42919.250} \\
\hline GA341 & \multicolumn{3}{|c|}{1.00} \\
\hline
\end{tabular}

Table 3: Result of RAMPAGE and MODELLER modules.

After the loop refinement and quality assessment using MODELLER, the final model was verified by Structural Analysis and Verification Server (SAVES) to evaluate its stereo-chemical quality. Ramachandran plot computed by PROCHECK module and other features of the structures were analyzed by different structure prediction servers. Following (Figure 4) are the results of final model using different programs. 
A

Overall modei quality

Z-Score: $\mathbf{- 3 . 8 9}$

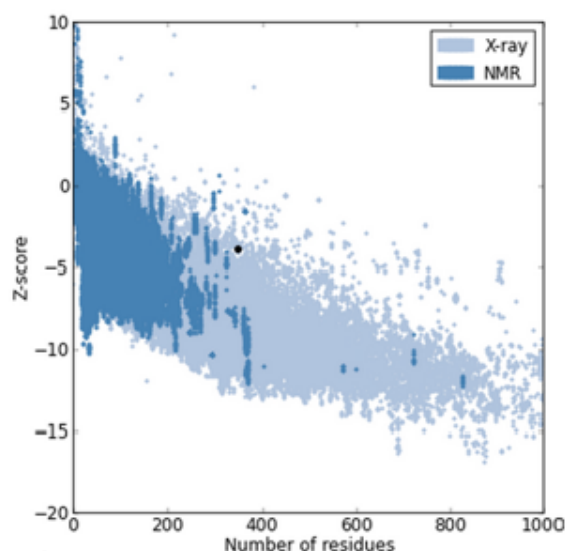

C

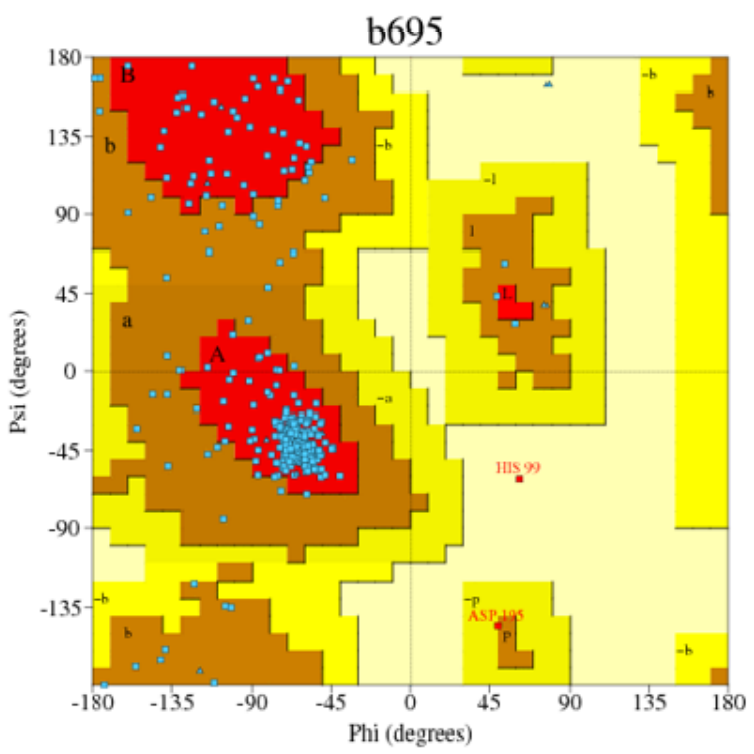

Program: ERRAT2

File:/var/www/SAVES/Jobs/32264419//errat.pdb

Chain\#:1

Overall quality factor*: 90.323

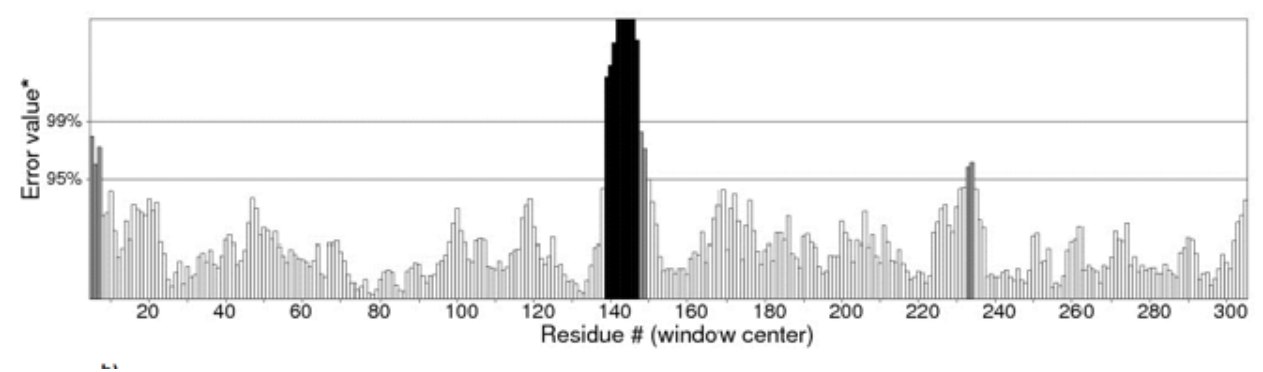

\section{Ramachandran Plot statistics}

\begin{tabular}{|c|c|c|c|}
\hline & & $\begin{array}{l}\text { No. of } \\
\text { residues }\end{array}$ & 8 -tage \\
\hline Most favoured regions & {$[A, B, L]$} & 271 & $85.2 \%$ \\
\hline Additional allowed regions & {$[a, b, 1, p]$} & 45 & 14.28 \\
\hline Generously allowed regions & {$[\sim a, \sim b, \sim 1, \sim p]$} & 1 & $0.3 \%$ \\
\hline Disallowed regions & {$[\mathrm{xx}]$} & 1 & $0.3 \% *$ \\
\hline Non-glycine and non-proline & residues & 318 & 100.08 \\
\hline End-residues (excl. Gly and & Pro) & 2 & \\
\hline Glycine residues & & 14 & \\
\hline Proline residues & & 16 & \\
\hline Total number of residues & & 350 & \\
\hline
\end{tabular}


Figure 4: a). ProSAweb result shows the overall quality of final structure. (b). Errat result by SAVES (Structural Analysis and Validation Server) results. c). Ramachandran plot by PROCHECK.

Evaluation of model-4 structural quality with ProSA-web (Figure 4a) revealed its ProSA Z-score value -3.89, falls in the range of native conformations computed using X-ray crystallography method represented as encircled as large black dot. Errat result shows the overall quality of the structure is 90.323 (Figure $\mathbf{4 b}$ ), which is very good experimentally and computationally. Ramachandran plot computed by PROCHECK module (Figure 4c) showed only $0.3 \%$ of residues exist in disallowed regions confirming the quality of $0.3 \%$ residues predicted to be highly significant.

\subsection{Molecular dynamics simulation of C5aR1:}

The resulting plots of each stage of MD simulation are represented as below: 
A

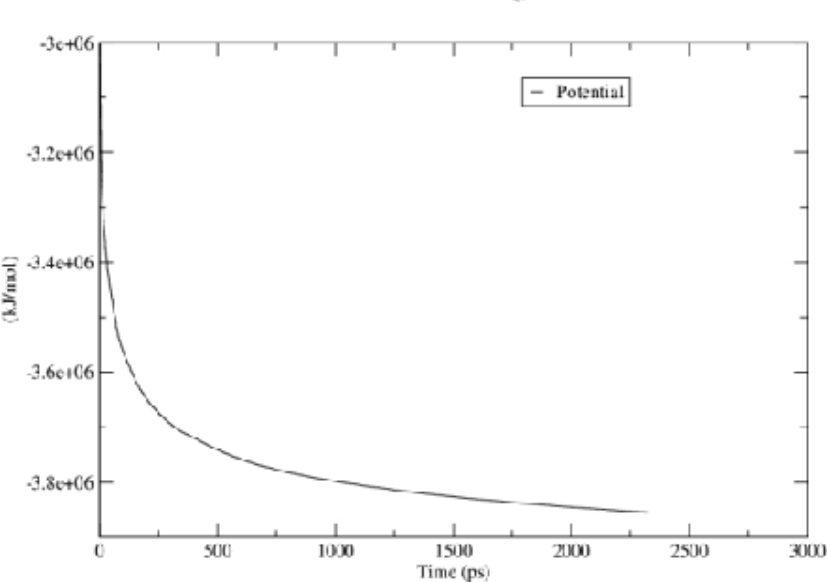

c

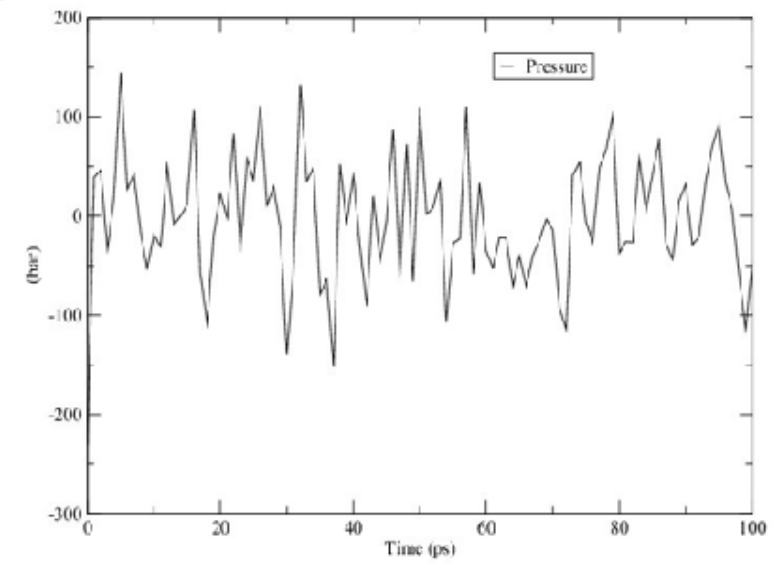

B

Gromacs Energies

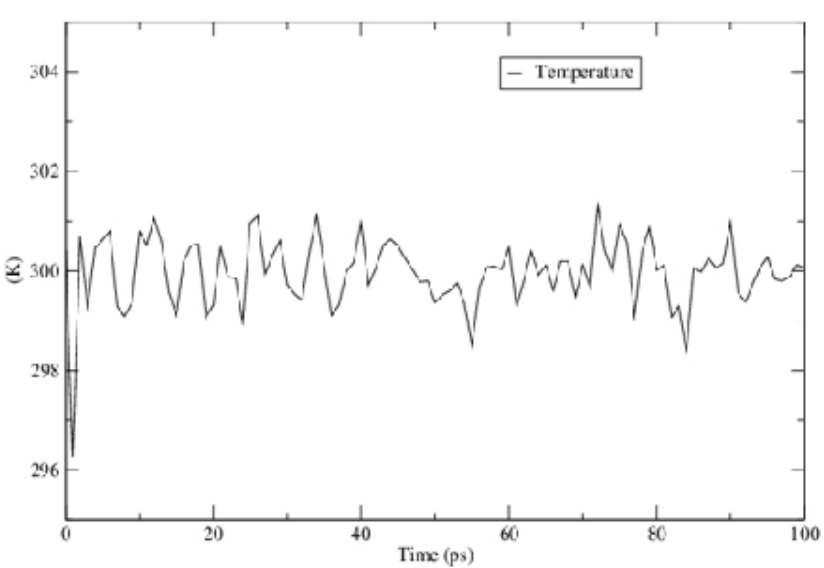

D

Gromacs Energies

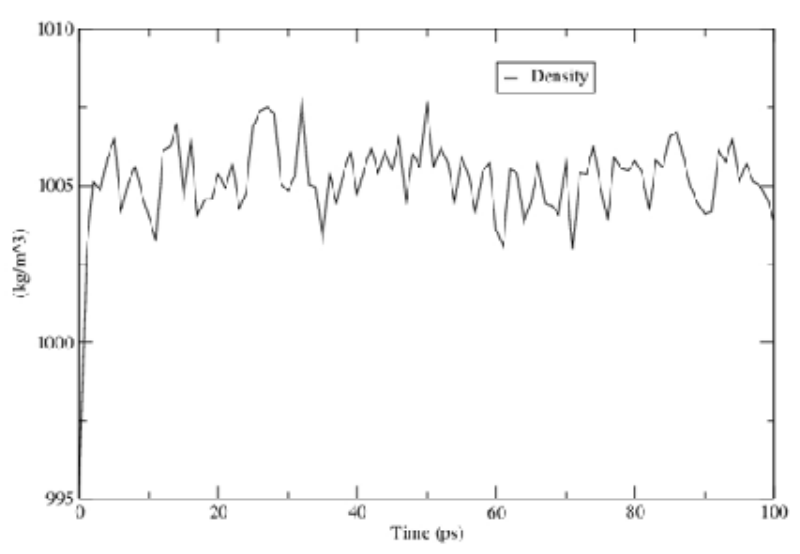

Figure 5: Parameters during MD simulation A) Energy minimization B) Temperature C) 


\section{Pressure D) Density plot}

From the above figures (Figure 5A) The Energy graph of Potential demonstrating steady convergence of $\mathrm{E}_{\mathrm{pot}}$ and from the temperature plot (Figure 5B), it is clear that the temperature of the system quickly reaches the target value (300K), and remains stable over the remainder of equilibration. From the Pressure plot (Figure 5C), pressure value fluctuates widely over the course of 100-ps equilibration phase, but this behavior is not unexpected and from the density plots (Figure 5D), we can clearly see that density values are very stable over time, indicating that the system is well-equilibrated now with respect to pressure and density.

A

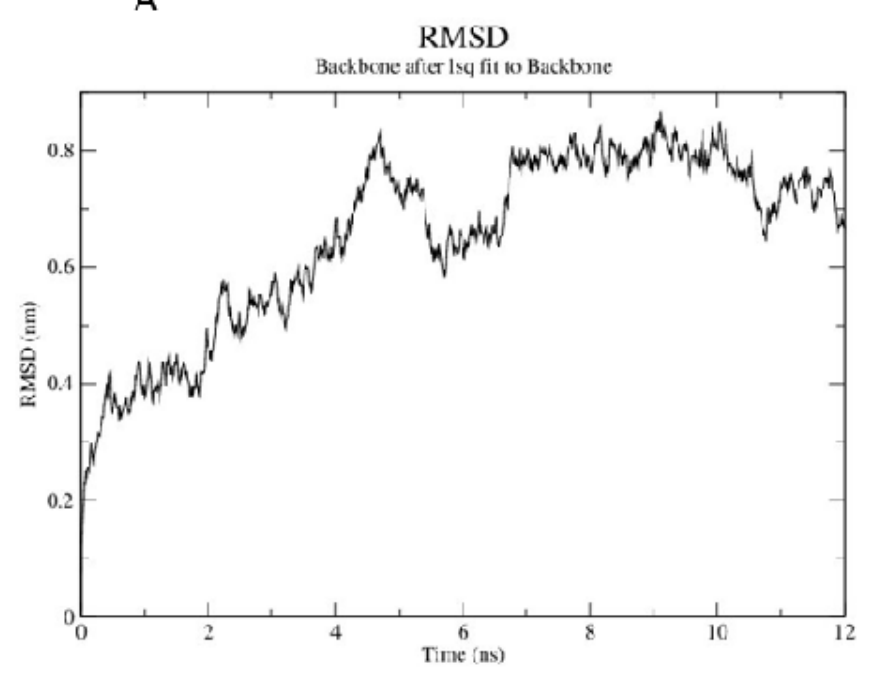

B

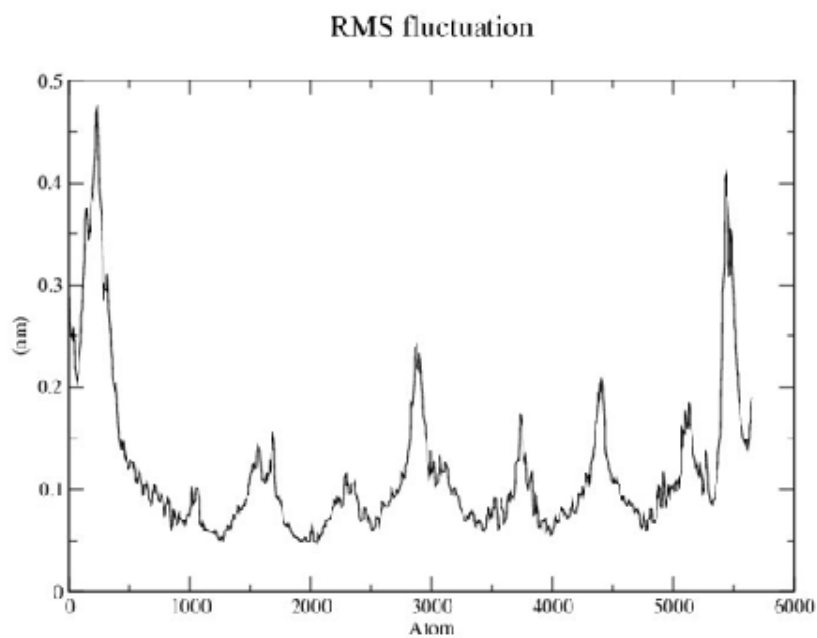

Figure 6: A) RMSD plot

B) RMSF plot 
Here, above the first figure (Figure 6A) shows all-atom RMSD of the modeled system against the time scale. After 6 ns time the system starts stabilizing, it can be visualized that after 6 ns the deviation was found to occur within a range of $0.7-0.8 \square$ nm, means within $1 \square \AA$, it can be regarded as stable. The second plot (Figure 6B) shows the root mean square fluctuation (RMSF) for each atom. It also shows flexibility for each atom but at some point (i. e. atom no. 3000), it gets fluctuated. 
(a)

\section{C5AR1 lipid bilayer system}

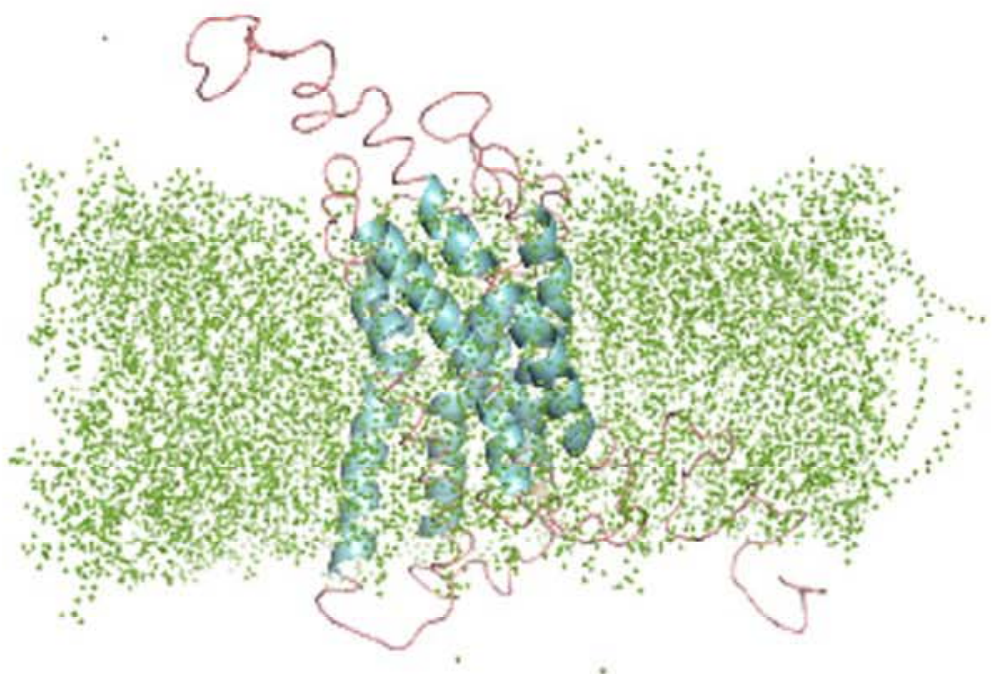

(c) Lipid bilayer MD RMSD Analysis

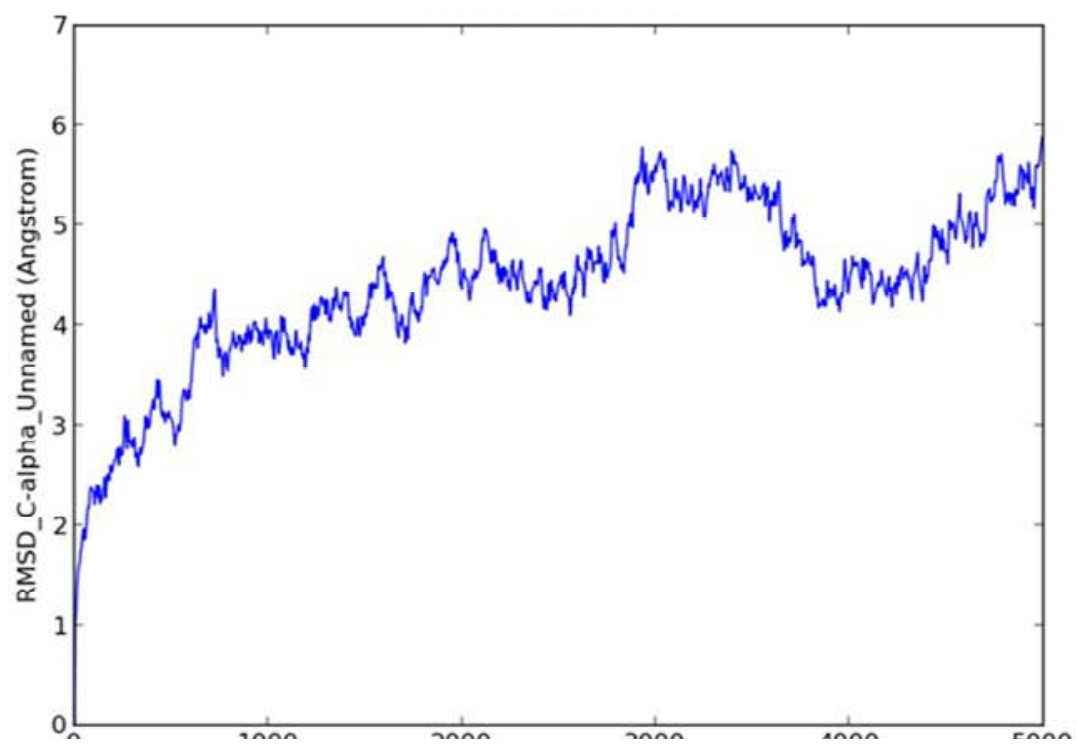

(b) Lipid bilayer MD Quality Analysis

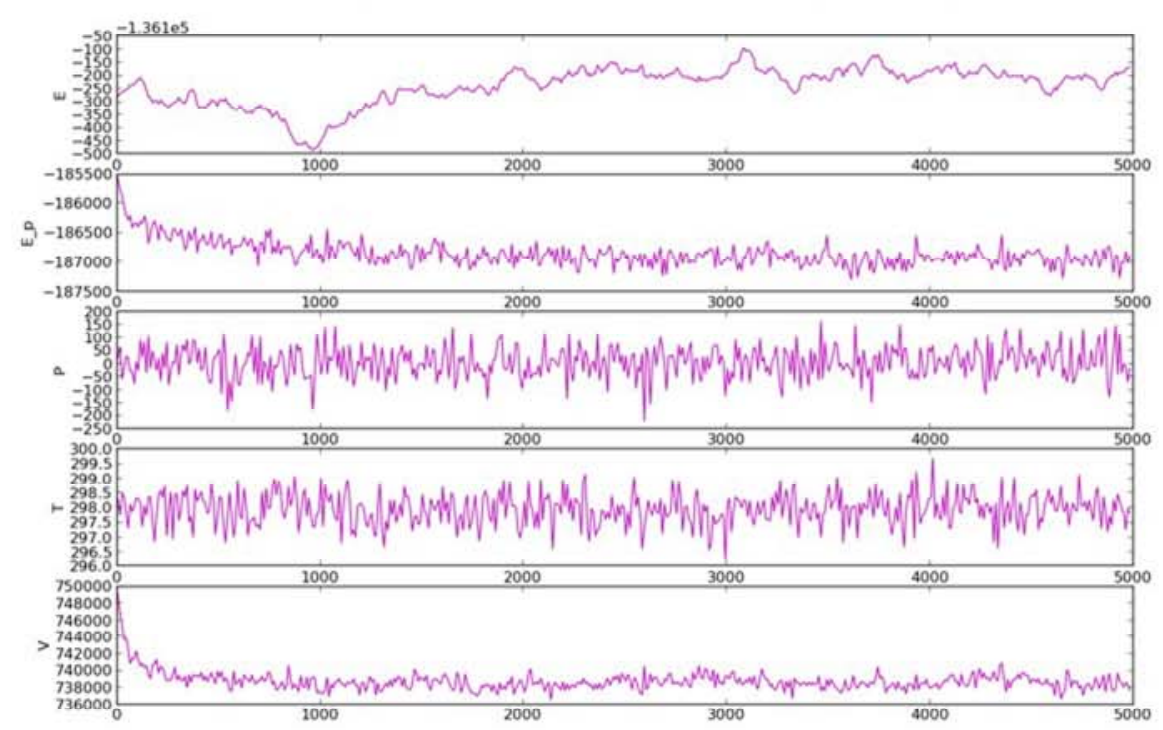

(d) Lipid bilayer MD RMSF Analysis

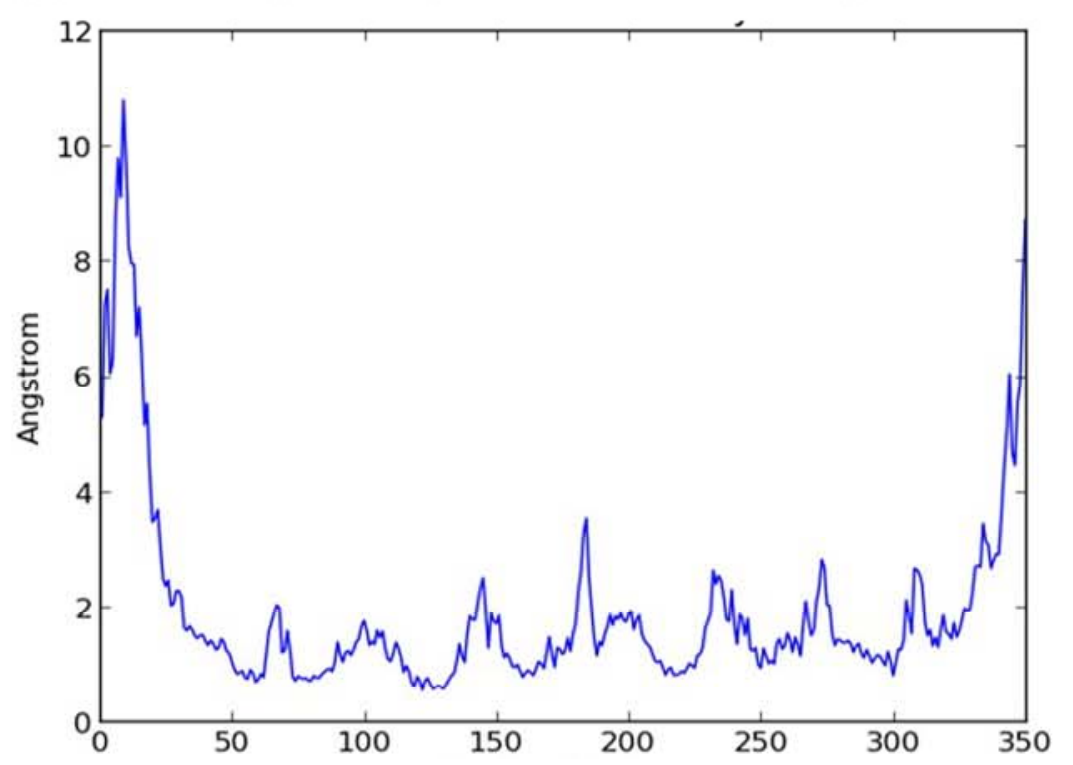


Fig. 7 a) C5aR1 Lipid Bilayer system (b) Quality plot generated for C5aR1(c) Lipid Bilayer MD RMSD analysis (d) Lipid bilayer MD RMSFanalysis 


\section{Discussion:}

In this study, we aimed to provide deeper insight into the species diversity of C5AR1 receptor genes of different groups. This study helped us to clarify some aspects of the evolutionary history of this interesting group. Based on the Bayesian inference phylogeny (Figure 1A), the Bayesian posterior probabilities (BPPs) values is almost 1 at every node, indicated that clade generated were the closest relatives among all the species used for the phylogenetic reconstruction. From the relaxed molecular clock (Figure 1B), it can be said that the clock is accelerating with respect to the out-group. The clades are highlighted with the different color in the final chronogram which confirmed that Rodentia, Artiodactyla, Primates, Carnivora and Reptilia form different clades according to their orders. The divergence times and the mean age estimates of most nodes were largely consistent. The phylogram gives the median age estimates for all nodes in the tree, which is million years. On the basis of chronogram (Figure 2), we can see that the oldest spilt in the tree is at around 63 Mya (million years ago). The molecular dating results indicate that clade formed for Rodentia (highlighted with blue) diverged approximately 29.61 mya (95\% Highest Posterior Density) from the other groups. It may be stated that within the Rodentia clade, the taxa Mus musculus and Rattus norvegicus diverged around 3.88 mya from each other, while clads highlighted with purple (Artiodactyla) and pink (Primates) diverged at 14.1 mya. The taxa within the group Carnivora and Reptilia diverged at approximately 12.64 and 32.79 mya respectively. Divergence among the individuals of six species (Homo sapiens, Pan paniscus, Gorilla, Pan troglodytes and Maccaca mulatta) were between 0.26 mya, 1.2 mya and 2.3mya respectively. The developed model of C5aR1 shows overall good structural quality and was confirmed using several different validation tools. It can act as a good chemo attractant target as $\mathrm{C} 5 \mathrm{a}$ interacts with receptor protein $\mathrm{C} 5 \mathrm{a} 1$ or $\mathrm{C} 5 \mathrm{aR}$ on the surface of target cells such as macrophages, neutrophils and endothelial cell. In Leishmania infection, the amastigote form of the pathogen is found present in human macrophages. So by targeting this C5AR receptor which is one of the most potent inflammatory chemoattractant and has been implicated in pathogenesis of numerous inflammatory diseases, we can further stop the cell division of amastigote form of Leishmania. Our study provides the molecular model and its in-silico structural stability but still further study needs to be done in order to understand the biochemical nature of the molecule. $\mathrm{C} 5 \mathrm{aR} 1$ has raised the intriguing possibility of the use of this receptor having possible role in inflammation. 


\section{Acknowledgement}

The authors would like to thank Department of Biotechnology, Government of India for intramural fund.

\section{References:}

1. Patrick Joost and Axel Methner. Phylogenetic analysis of 277 human G-protein-coupled receptors as a tool for the prediction of orphan receptor ligands Patrick Joost and Axel Methner. Genome Biology (2002), 0063.1-0063.16.

2. Manthey HD, Woodruff TM, Taylor SM, Monk PN. Complement component 5a (C5a). Int J Biochem Cell Biol (2009) 41 (11).

3. Hani Boshra, Jun Li, Rodney Peters, John Hansen, Anjan Matlapudi and J. Oriol Sunyer. Cloning, Expression, Cellular Distribution, and Role in Chemotaxis of a C5a Receptor in Rainbow Trout: The First Identification of a C5a Receptor in a Nonmammalian Species. The Journal of Immunology (2004), 4381-4390.

4. Gerard, N. P., and C. Gerard. The chemotactic receptor for human C5a anaphylatoxin. Nature (1991), 349-614.

5. Bachvarov, D. R., S. Houle, M. Bachvarova, J. Bouthillier, S.A. St. Pierre, Y. Fukuoka, J.A. Ember, and F. Marceau. Clonning and preliminary pharmacological characterization of the anaphylatoxin C5a receptor in the rabbit. Br. J. Pharmacol (1999), 128-321.

6. Hartley, M. A., Kohl, K., Ronet, C., \& Fasel, N. (2013). The therapeutic potential of immune cross-talk in leishmaniasis. Clinical Microbiology and Infection, 19(2), 119-130.

7. Larkin MA, Blackshields G, Brown NP, Chenna R, McGettigan PA, McWilliam H, Valentin F, Wallace IM, Wilm A, Lopez R, Thompson JD, Gibson TJ, Higgins DG. Clustal W and Clustal X version 2.0. Bioinformatics (2007), 2947-2948.

8. Huelsenbeck J. P., Ronquist F., Nielsen R., Bollback J.P. Bayesian inference of phylogeny and its impact on evolutionary bioilogy. Science (2001), 2310-2314.

9. Huelsenbeck J. P., Ronquist F. MrBayes: Bayesian inference of phylogenetic trees. Bioinformatics (2001). 754-755. 
10. Morariu, V.I., Srinivasan, B. V., Raykar, V.C., Duraiswami, R., Davis, L.S. Automatics online tuning for fast Gaussian summation. In Advance in Neural Information processing systems (2009). 1113-1120.

11. Ambrish Roy, Alper Kucukural \& Yang Zhang, I-TASSER: a unified platform for automated protein structure and function prediction. Nature Protocols (2010), 5:725-738.

12. Laskowski RA, Hutchinson EG, Michie AD, Wallace AC, Jones ML, et al. PDBsum: a Web-based database of summaries and analyses of all PDB structures. Trends Biochem Sci (1997) 22: 480-490

13. Wiederstein M, Sippl MJ. ProSA-web: interactive web service for the recognition of errors in tree-dimensional structures of proteins. Nuc Acids Res (2007) 407-410

14. Laskowski RA, Macarthur MW, Moss DS, Thornton JM PROCHECK: A program to check the stereochemical quality of protein structures. J Appl Crystallogr (1993) 26: 283-291.

15. Sander Pronk, Szilárd Páll, Roland Schulz. Gromacs 4,5: a high-throughput and highly parallel open source molecular simulation toolkit. Bioinformatics (2013) ; 29(7): 845854.

16. The PyMol Molecular Graphics System, Version 1.3, Schrodinger, LLC. 



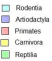

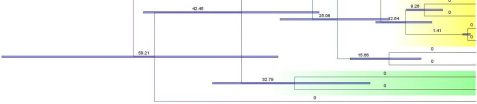




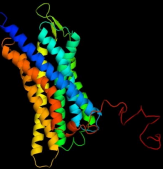




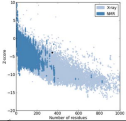

\section{Progan trukT:}

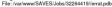

Chaiks 1

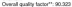
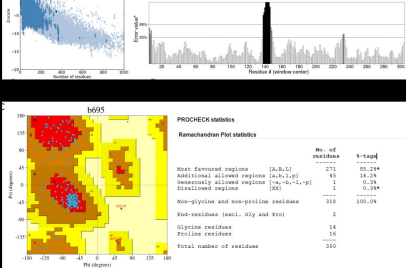

PHocreck vesabes

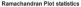

\begin{tabular}{|c|c|c|c|}
\hline & & $\begin{array}{l}\text { Ho, of } \\
\text { meaidzane }\end{array}$ & $4-t a g d$ \\
\hline Staet favoured segienn & {$[\mathrm{A}, \mathrm{D}, \mathrm{B}]$} & 271 & as .21+ \\
\hline Additiunal at Jowd zepiens & ta,bil,pl & 43 & 14.24 . \\
\hline Deneveusiy aljored soghana & {$[-a,-b,-1,-p]$} & 1. & 0.25 \\
\hline 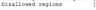 & $|x \times|$. & $i$ & $0.95+$ \\
\hline Xanjlycinn and nanrotine & Imiduen & 318 & 100,04 \\
\hline Ent-ressous lemot, gif as & reol & 2 & \\
\hline olsctre zadtcea & & 14 & \\
\hline Deslema zarides & & it & \\
\hline 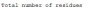 & & aso & \\
\hline
\end{tabular}




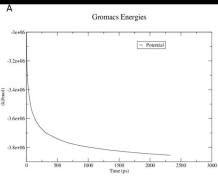

Gromacs Energie

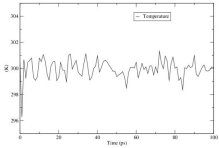

Gimeises Energies

D

Gromacs Eacrgies
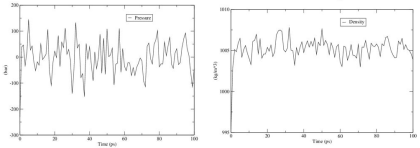
RMSD

Eudtrens ahar Inq feis liaddeas

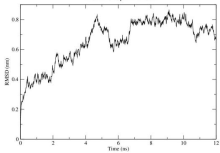

RMS fluctiation

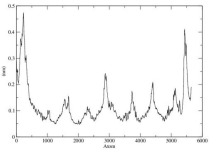


(a) C5AR1 lipid bilayer system

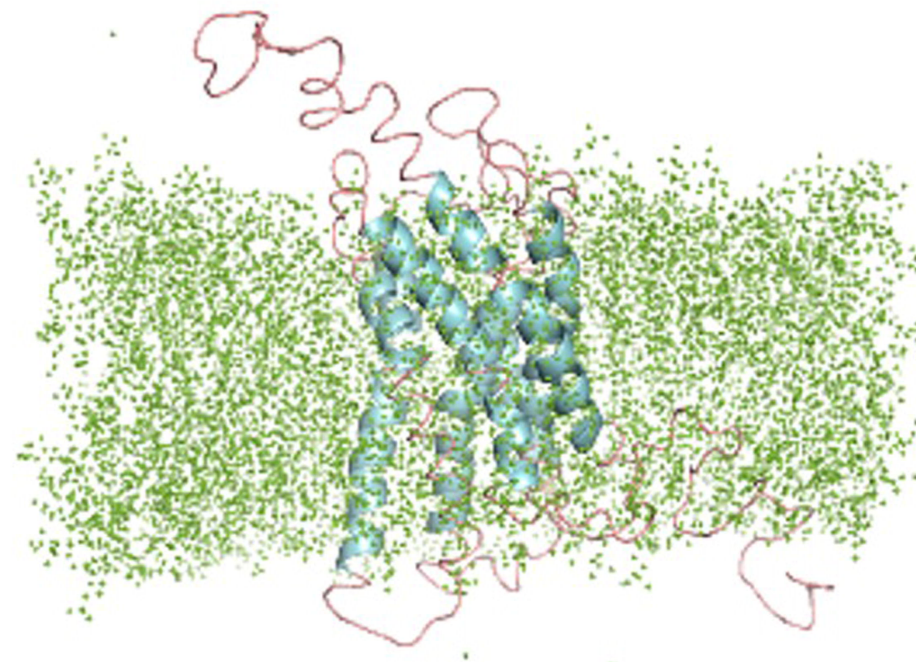

(c) Lipid bilayer MD RMSD Analysis

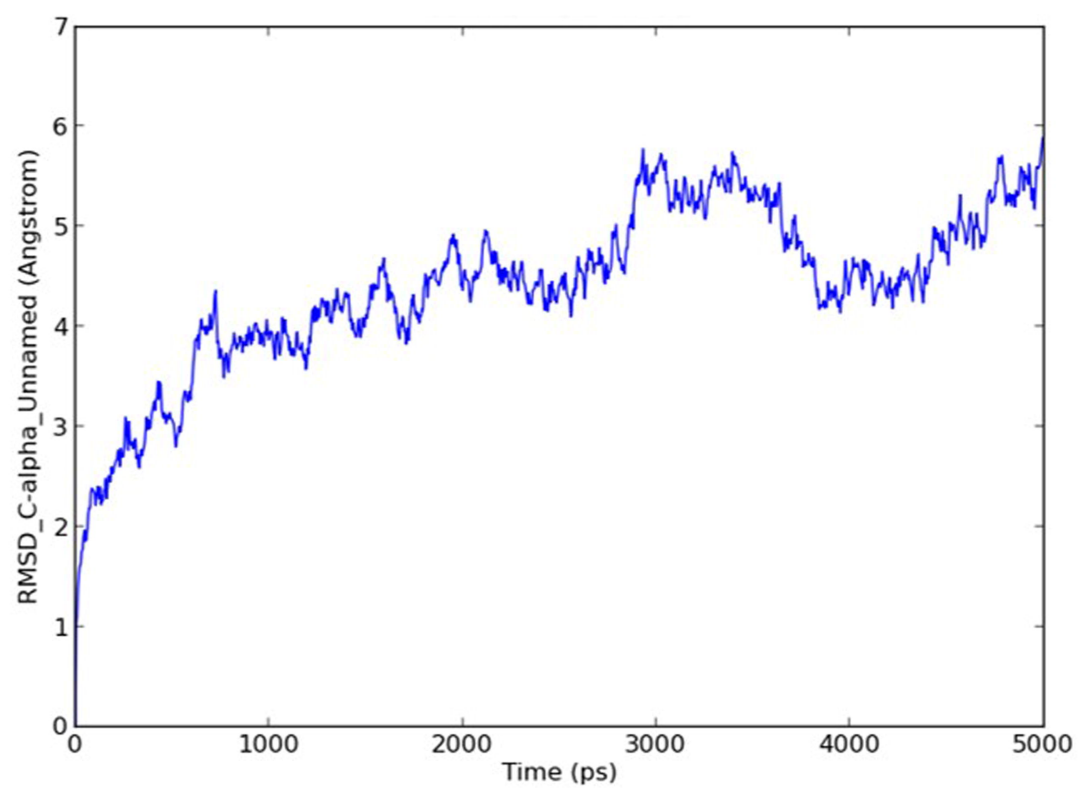

(b) Lipid bilayer MD Quality Analysis

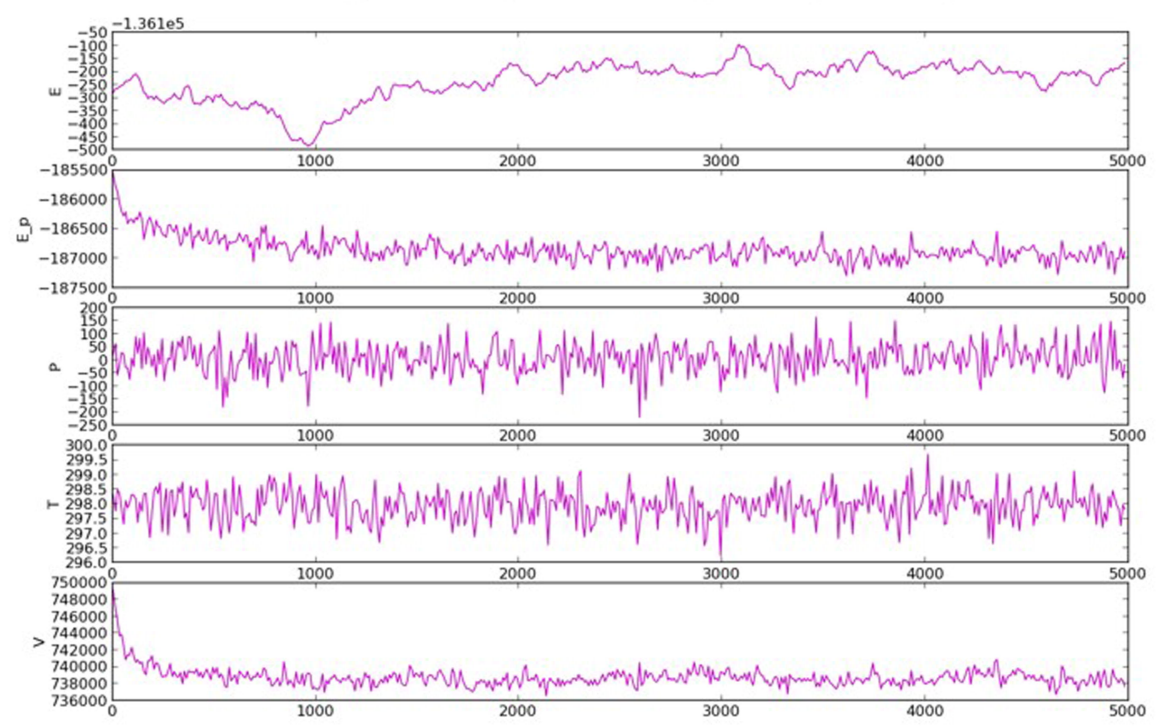

(d)

Lipid bilayer MD RMSF Analysis

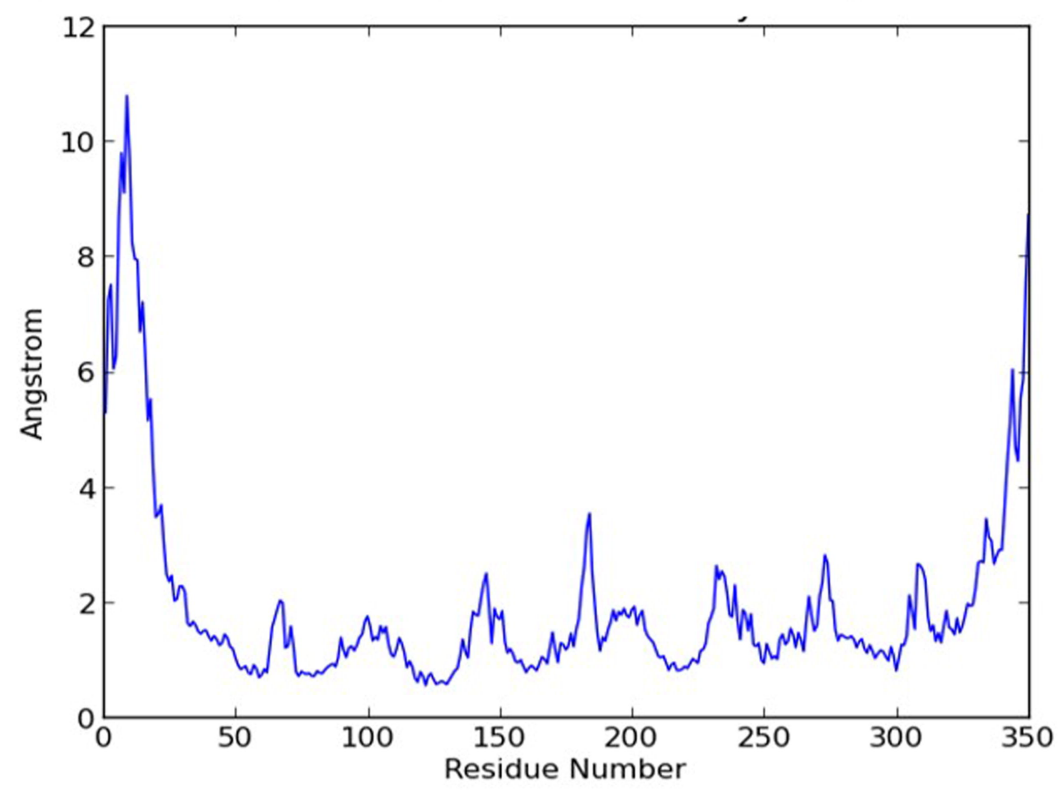

